\title{
Epitheliopathy of the bleb (EoB)-identifying attrition: A new model for failure of glaucoma surgery
}

\author{
Gysbert-Botho van Setten ${ }^{1,2 *}$ \\ ${ }^{1}$ Department of Clinical Neuroscience, St Eriks Eye Hospital, Karolinska Institutet, Sweden \\ ${ }^{2}$ Department of Ob/Gyn, Institute of Wound Research, University of Florida, Gainesville, FL, USA
}

\begin{abstract}
Introduction: Fluorescein staining of the ocular surface is used to detect anomalies and damage of the ocular surface. The intensity of staining and its pattern does reflect underlying pathology and severity. We have recently reported on the presence of sandbank epitheliopathy and its specific staining of the conjunctival surface. As glaucoma surgery is known to alter ocular tissues structures we investigated if it might cause a characteristic footpint at the ocular surface.

Materials and methods: Fluorescein staining was routinely performed in all patients having undergone filtration surgery, both trabeculectomy and deep sclerectomy; directly after surgery as well as during the months following surgery. Slitlamp photography was conducted using a cobalt blue filter and a yellow filter within 30 seconds after application of a standardized amount of fluorescein. The results were compared to the recently described features of anatomical dry eye and sandbank epitheliopathy.

Results: In the weeks following surgery, the initially visible fluorescein staining close to the sutures and wound edge rapidly decreased. With the stabilization of the bleb, however, in large number of eyes specific staining for fluorescein prevailed that did not entirely disappear even weeks after surgery. Once the bleb, visible sign of functionality of filtration efficacy, had stabilized, specific staining was still observed in many patients on the surface of the bleb months after the surgery. This characteristically localized and mostly punctate staining, hereinafter defined as epitheliopathy of the bleb (EoB), occurred most often towards the limbal side of the bleb. On the side of the bleb towards the fornixes the staining was usually less and had mostly a sandbank epitheliopathy like appearance of low intensity. Such staining occurred mainly when tear fluid insufficiency was simultaneously present.
\end{abstract}

Discussion: It has now commonly been recognized that surgery does alter the ocular surface in that dry eye symptoms may be provoked. However, these changes are considered usually as temporary and with that it's patho-physiological secondary effects are considered less important. This is different with glaucoma surgery, when filtration surgery is concerned. The principle of this surgery consists in the creation of a filtration sides underneath the conjunctiva or tenon, visible as filtration bleb. It is the functionality of this filtration site that does decide of a successor or failure of the entire procedure. Functionality can only be maintained if wound healing is effectively silenced. Visible staining of the epithelium at or over the filtration site, however, does reflect localized injury, stress and ongoing healing processes, indicating secondary activity in the subconjunctival space. This could trigger stimulation of fibrosis leading to failure of filtration.

Conclusion: Fluorescein staining on the bleb, reflecting localized epitheliopathy is of major diagnostic importance and needs to be therapeutically appropriately addressed. Epitheliopathy of the bleb (EoB) is the result of friction is caused by attrition. Attrition is, per definition, the process of reducing something's strength or effectiveness through sustained attack or pressure. This exactly the case in the ocular surface of the surgically created protruding bleb, constantly exposed to the eroding forces of the environment and lid movement. Attrition may lead by mechanical stimulation to induction or continuation of fibrosis threatening the functionality of the bleb. Visible EoB could hence be a key indicator for the need of lubricating agents able to cope with the local enhanced rheological demands. Amongst the available agents, not unexpectedly, non-preserved gels and compositions containing high molecular weight hyaluronic acid formulations have been shown to be particularly suitable.

\section{Introduction}

A filtration bleb is the most common visible result of glaucoma surgery in the attempt to regulate of elevated intraocular pressure by filtration surgery. Easily accessible for inspection, its form, size and extension does vary considerably and there are different opinions about the optimal location, shape and dimensions. All in common is the threat of post-operative scar formation in these areas. Scar formation, i.e. fibrosis of the filtering tract or site is one of the main reasons for failure of glaucoma filtration surgery. The failure rate within 2 years was reported to be just below $20 \%$ [1]. The risk for fibrosis begins with the creation of the wound and the passage of anterior chamber fluid into an artificial cave located under the conjunctiva or tenon. Although the intensity of tissue reaction following surgery, may vary - depending on the surgical technique used, surgery itself causes activation of cells, followed by proliferation of fibroblasts and extracellular matrix (ECM) formation. These are processes in which transforming growth factor
(TGF-b1) and connective tissue growth factor (CTGF) are key actors $[2,3]$, wherein CTGF serves as downstream mediator of TGF-beta for fibroblasts [4]. It has been shown that fibrosis causing the failure of surgery did not differ immunohistochemically between trabeculectomy or Ahmed valves, suggesting a similar mechanism [5]. Specific genes involved in this process have been identified [6]. Also does the fluid entering the drainage cavity contain various cytokines [7] and could herewith contribute to initiation and maintenance of fibrosis of the

*Correspondence to: Gysbert -Botho van Setten, Department of Clinical Neuroscience, St Eriks Eye Hospital, Karolinska Institutet, Polhemsgatan 50, 11282 Stockholm, Sweden, E-mail: gysbert-botho.vansetten@sankterik.se

Key words: glaucoma surgery, ocular surface, fibrosis, fluorescein staining, bleb, epithelium

Received: April 06, 2018; Accepted: April 18, 2018; Published: April 20, 2018 
bleb. They may be called the internal effectors influencing the future of the filtration bleb. Then, there are others forces who that may trigger and maintain fibrotic events coming from the outside of the exposed surface influencing the space under the epithelium. One of such factors is CTGF which is upregulated by stretching forces $[8,9]$, although the exact molecular mechanism by which this occurs are not entirely clarified. Mechanical forces, potentially triggering fibrosis, may additionally be expected when the lid constantly moves over the bleb. Signs of such mechanical attrition has been recently reviewed for the lid side, focusing on lid wiper epitheliopathy [10]. Here the pressure of the lid on the eye is about $20 \mathrm{mmHg}$ in eyes without LWE and higher in those with LWE. When such forces are continuously and with every lid movement repeatedly exerted to an area of anatomical protrusion such as the bleb, they could, possibly, influence the ocular surface in this area. Hence, if mechanical stimulation could threaten the surgical result, clinical clues to identify areas of such mechanical irritation are very important. Therefore, the bleb of patients having had glaucoma surgery for was investigated changes on the conjunctival epithelium, both for identifying their presence and location but also in regard of their potential suitability to identify success of treatment as sign of clinical improvement.

\section{Materials and methods}

Fluorescein staining was routinely performed in all patients having undergone filtration surgery, both trabeculectomy and deep sclerectomy; directly after surgery as well as during the months following surgery. Slitlamp photography was conducted using a cobalt blue filter and a yellow filter within 30 seconds after application of a standardized amount of fluorescein. The results were compared to the recently described features of anatomical dry eye and sandbank epitheliopathy $[11,12]$.

\section{Results}

In the weeks following surgery, the initially visible fluorescein staining close to the sutures rapidly decreased. With decreases of size of the bleb also the staining does diminish remarkably during the first weeks of surgery (Figure 1).

With the formation of the bleb, however, in large number of eyes specific staining for fluorescein did not entirely disappear. Once the bleb, visible sign of functionality of filtration efficacy, had established, specific staining was observed on the surface of the bleb. Localized and mostly punctate staining occurred most often towards the limbal side of the bleb and could even extend over the corneal periphery (Figure 2).

On the side of the bleb towards the fornices the staining was usually less and had mostly a sandbank epitheliopathy $[11,12]$ like appearance. Such staining occurred mainly when tear fluid insufficiency was simultaneously present. The main area with fluorescein staining was often observed over the highest part of the bleb and on the downhill side of the bleb towards the limbus, i.e. close to the maximum slope of the bleb, there where friction and stress was considered to be strongest (Figure 3).

\section{Discussion}

It was shown that specific fluorescein staining does occur in the area of the bleb, mainly on the limbal side of the bleb, indicating enhanced localized ocular surface stress. This staining, as different to other staining patterns found in anatomical dry eye and sandbankepitheliopathy is, in distinction to them, now named Epitheliopathy of the Bleb (EoB) and does co-localize with the areas of enhanced tissue stress caused by the anatomical protusion and pressure of the moving eye lid. This stress, imposing repeated and sustained attack or pressure

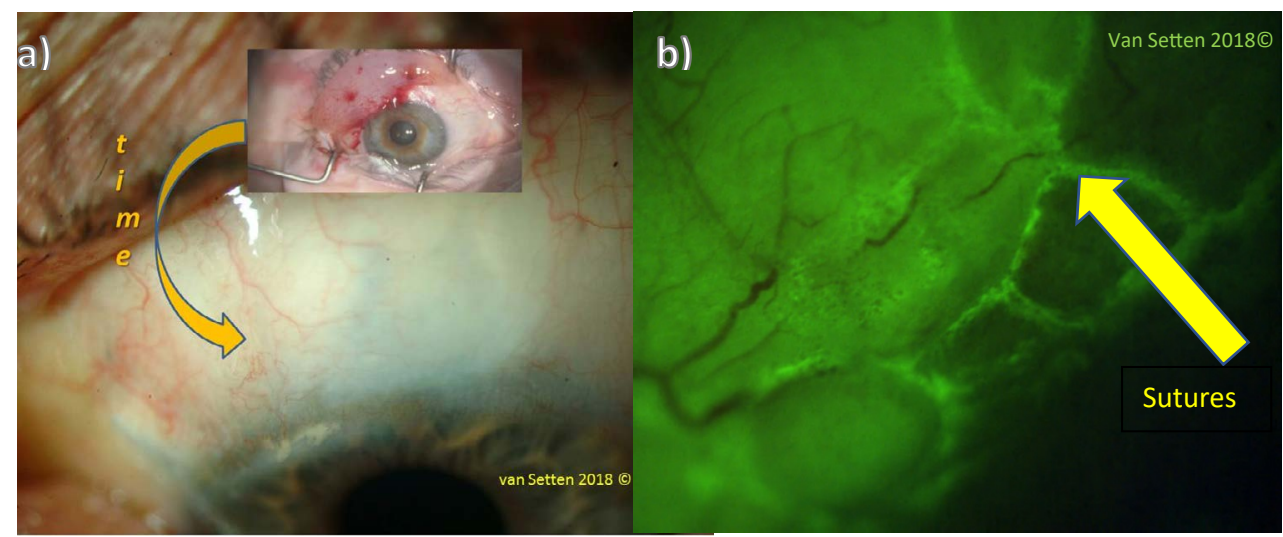

Figure 1. a) Decrease of the elevation of the bleb after deep sclerectomy within a few weeks after surgery, b) Localized staining at the site of sutures after glaucoma surgery.

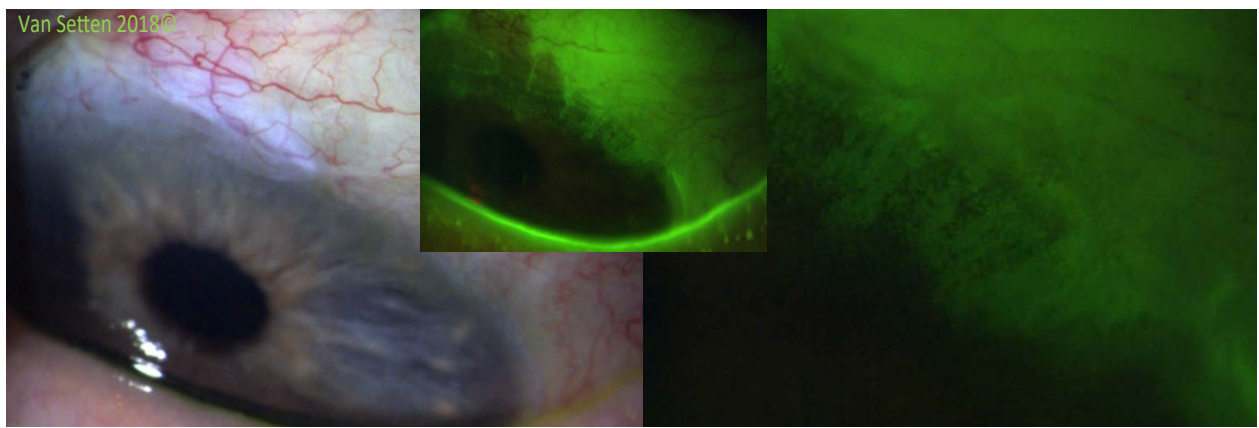

Figure 2. Staining at the limbal side of the bleb, extending over the corneal periphery 

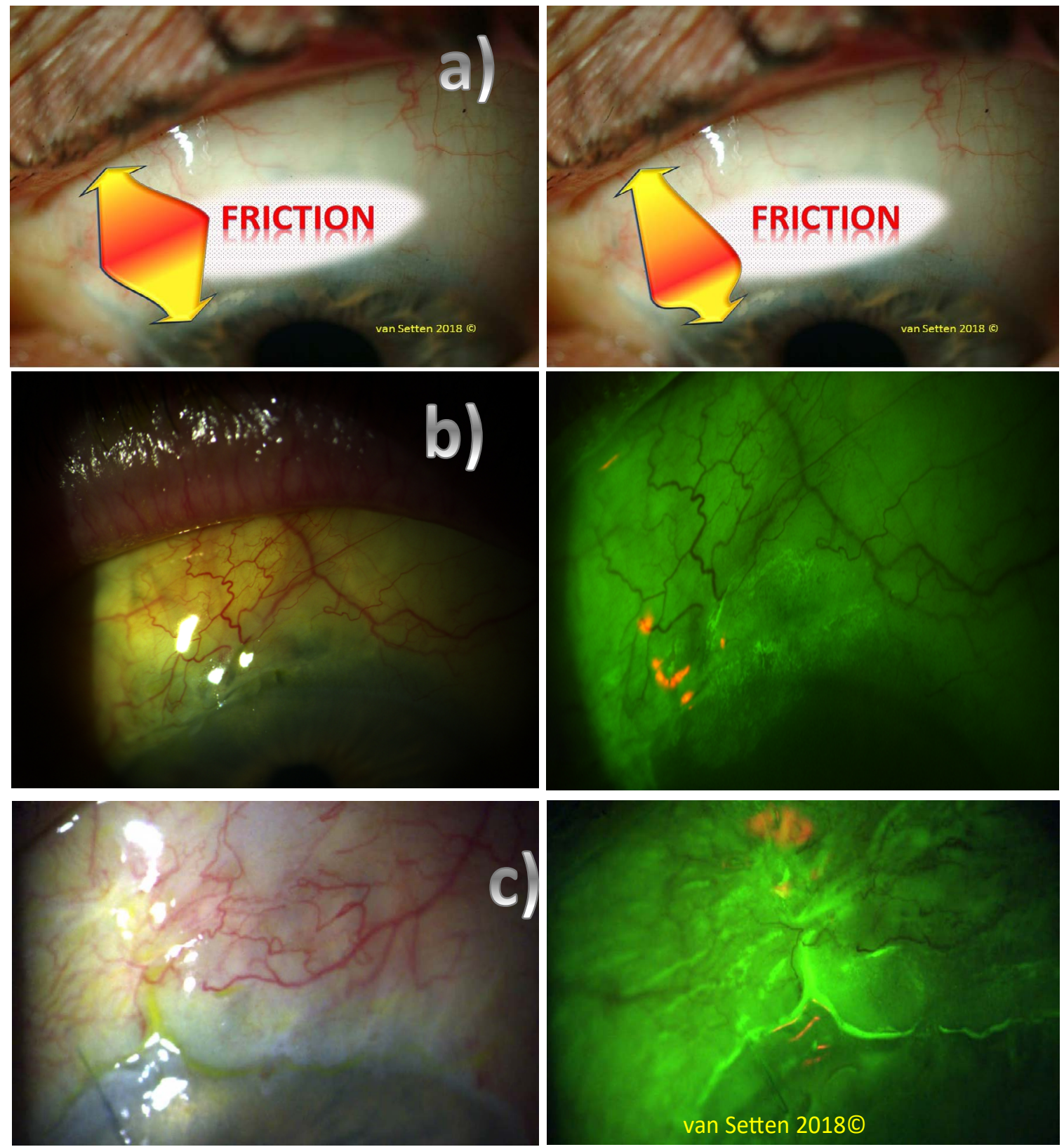

Figure 3a. Location of main friction areas in the area of the bleb (schematic); 3b. Localized punctate staining at the downhill side of the bleb towards the limbus; 3c. Localized staining over a cystic area of the bleb

on the often-thin conjunctiva covering the bleb does create attrition. Attrition is, per definition, the process of reducing something's strength or effectiveness. These effects reach under the surface, whilst the visibility is dependent on staining. As shown above, the clinical appearance alone without staining does not provide and reliable clue about the presence of localized surface anomalies. The only clue is provided by staining with vital dyes, such as fluorescein. In areas identified by staining as EoB, attrition may be expected, capable of leading via mechanical stimulation to induction or continuation of fibrosis threatening the functionality of the bleb which is a complicated process [13]. On the other side attrition may lead to thinning of the conjunctiva covered by a so called attenuated epithelium [14]. In these areas the presence and action of metalloproteases may have decisive role considering survival of the bleb [15]. Additionally, they come with the risk of thinning of the bleb wall due to apoptosis and localized eradication of any wound healing response. In general, however, is the observed accumulation of latent transforming growth factor $\beta$ in the epthelium of the bleb [16] of major importance since CTGF, downstream mediator of TGF- $\beta$ is a major actor in scarformation $[17,18]$. It is hence these, the secondary effects, provoked by attrition in tissues with high capacity of tissue regeneration, that can lead to failure of the bleb. It is well known that the healing ambition of the tissues surrounding the surgically created sub conjunctival space can trigger fibrotic events. This is the therefore the domain for the use of anti-metabolites, mainly Mitomycin $\mathrm{C}$ and $5 \mathrm{FU}$. However, even the effects of these substances as cell response silencers are temporarily limited. The majority of cases of glaucoma surgery areas will, eventually, face the risk of progressively increasing fibrotic events leading finally to the failure of the filtration site. The 
triggering stimuli that help to maintain fibrotic stimulation seem to be indeed primarily mechanical by nature. A major force is the pressure of the eyelid, rubbing and stretching the surface continuously. The resulting physical correlating parameter is suspected to be friction. Friction does increase cell response such like to CTGF release, trigger the inflammatory cascade and with that at the end fibrotic events. Under these circumstances, it seems obvious that attenuation of friction is the main targets of postoperative treatment following the trading glaucoma surgery. However, the continuous use of lubricating agents, especially in eyes having yet no visible signs of dry eye disease [19], is today not so well recognized, especially as osmolarity does not play a major role in this localized area of stress. Here again, aside from the need to understand for the necessity for therapy, it must be emphasized that special requirements do apply for the physical chemical parameters characterizing the substances used. Obviously simple watery teardrops with their decreased capacity of influencing tear viscosity or even reducing the viscosity of the remaining tears are not the therapy of choice. Substances used to decrease friction in these artificially enhanced exposed areas, i.e. the bleb, should be searched in the area of gels and ointments. Here the galenic features and composition is decisive for the desired functionality, i.e. decreasing friction. In the case of EoB the demands on fluid characteristics are apparently different from those reviewed for the ocular surface in "common" dry eye disease (Tiffany 1991). Initial results have shown that the treatment with viscous, preservative-free agents, preferentially non-Newtonian agents such as high molecular weight hyaluronic acid have the capacity to visibly reduce the fluorescein staining at the surface indicating attenuation of attrition of the bleb. Most important aspect within this treatment is the absence of potentially or inherently toxic preservatives.

\section{Conclusion}

Epitheliopathy of the bleb (EoB) should be actively looked after following any glaucoma filtration surgery because it is a key indicator of localized imbalance of epithelial homeostasis and attrition, identifying areas of enhanced mechanical stress, threatening to activate and maintain continuous wound healing of the bleb and therefore emphasizing the need of lubricating agents able to cope with the local rheological demands. Here, high molecular weight hyaluronic acid formulations appear to be particularly suitable.

\section{Acknowledgment}

The author is very thankful to the FoUU forskningsfond of the Karolinska Institutet,Stockholm supporting with the costs of publication.

The technical support of Aviation-Ophthalmology, Stockholm, Sweden is thankfully acknowledged.

\section{References}

1. Matlach J, Hipp M, Wagner M, Heuschmann PU, Klink T, et al. (2015) A comparative study of a modified filtering trabeculotomy and conventional trabeculectomy. Clin Ophthalmol 9: 483-492. [Crossref]
2. Chen N,Guo D,Guo Y,Sun Y, Bi H, et al. (2016) Paclitaxel inhibits cell proliferation and collagen lattice contraction via TGF-b signaling pathway in human Tenon's fibroblasts in vitro. Eur J Pharmacol 777: 33-40.

3. Van de Velde S, Van Bergen T, Vandewalle E, Kindt N, Castermans K, et al. (2015) Rho kinase inhibitor AMA0526 improves surgical outcome in a rabbit model of glaucoma filtration surgery. Prog Brain Res 220: 283-297. [Crossref]

4. Grotendorst GR (1997) Connective tissue growth factor: a mediator of TGF-beta action on fibroblasts. Cytokine Growth Factor Rev 8: 171-179. [Crossref]

5. Bae K, Suh W, Kee C (2012) Comparative study of encapsulated blebs following Ahmed glaucoma valve implantation and trabeculectomy with mitomycin-C. Korean J Ophthalmol 26: 265-270. [Crossref]

6. Mahale A, Othman MW, Al Shahwan S, Al Jadaan I, Owaydha O, et al. (2015) Altered expression of fibrosis genes in capsules of failed Ahmed glaucoma valve implants. PLoS One 10: e0122409. [Crossref]

7. Ecker SM, Hines JC, Pfahler SM, Glaser BM (2011) Aqueous cytokine and growth factor levels do not reliably reflect those levels found in the vitreous. Mol Vis 17: 2856263. [Crossref]

8. Chaqour B, Goppelt-Struebe M (2006) Mechanical regulation of the Cyr61/CCN1 and CTGF/CCN2 proteins. FEBS J 273: 3639-3649. [Crossref]

9. Hishikawa K, Oemar BS, Nakaki T (2001) Static pressure regulates connective tissue growth factor expression in human mesangial cells. J Biol Chem 276: 16797-16803. [Crossref]

10. Efron N, Brennan NA, Morgan PB, Wilson T (2016) Lid wiper epitheliopathy. Prog Retin Eye Res 53: 140-174. [Crossref]

11. van Setten G (2017a) Sandbank Epitheliopathy of the Conjunctiva (SEC) : A new indicator in dry eye diagnostics useful for optimized ocular surgery. J Eye Cataract Surg 3: 1-4

12. van Setten G (2017b)The Anatomical Dry Eye A Different Form of Ocular Surface Disease Deserves Focus. Open Journal of Ophthalmology 7: 184-190.

13. Van Bergen T, Vandewalle E, Van de Veire S, Dewerchin M, Stassen JM, et al. (2011) The role of different VEGF isoforms in scar formation after glaucoma filtration surgery. Exp Eye Res 93: 689-699. [Crossref]

14. Bouhenni RA, Al Jadaan I, Rassavong H, Al Shahwan S, Al Katan H, et al. (2016) Lymphatic and Blood Vessel Density in Human Conjunctiva After Glaucoma Filtration Surgery. J Glaucoma 25: e35-38. [Crossref]

15. Mathalone N, Marmor S, Rahat MA, Lahat N, Oron Y, et al. (2011) MMP expression in leaking filtering blebs and tears after glaucoma filtering surgery. Graefes Arch Clin Exp Ophthalmol 249: 1047-1055. [Crossref]

16. Saika S, Yamanaka O, Baba Y, Kawashima Y, Shirai K, et al. (2001) Accumulation of latent transforming growth factor-beta binding protein-1 and TGF beta 1 in extracellular matrix of filtering bleb and of cultured human subconjunctival fibroblasts. Graefes Arch Clin Exp Ophthalmol 239: 234-241. [Crossref]

17. Daniels JT, Schultz GS, Blalock TD, Garrett Q, Grotendorst GR, et al. (2003) Mediation of transforming growth factor-beta(1)-stimulated matrix contraction by fibroblasts: a role for connective tissue growth factor in contractile scarring. Am J Pathol 163: 20432052. [Crossref]

18. Esson DW, Neelakantan A, Iyer SA, Blalock TD, Balasubramanian L, et al. (2004) Expression of connective tissue growth factor after glaucoma filtration surgery in a rabbit model. Invest Ophthalmol Vis Sci 45: 485-491. [Crossref]

19. Craig JP, Nelson JD, Azar DT, Belmonte C, Bron AJ, et al. (2017) TFOS DEWS II Report Executive Summary. Ocul Surf 15: 802-812. [Crossref]

Copyright: (C)2018 Gysbert -Botho van Setten. This is an open-access article distributed under the terms of the Creative Commons Attribution License, which permits unrestricted use, distribution, and reproduction in any medium, provided the original author and source are credited. 